Research Journal of Microbiology 6 (2): 159-165, 2011

ISSN 1816-4935 / DOI: 10.3923/jm.2011.159.165

(C) 2011 Academic Journals Inc.

\title{
Frequencies of Salmonella enterica in Growing Pigs in Paraguay
}

${ }^{1}$ J. Copes, ${ }^{1}$ G. Leotta, ${ }^{2}$ L. Cardozo, ${ }^{2}$ G. Gimenez, ${ }^{2}$ L. Nuñez, ${ }^{2,3}$ N. Zarate, ${ }^{2}$ D. Lopez, ${ }^{3} \mathrm{~N}$. Weiler, ${ }^{3} \mathrm{M}$. Alvarez and ${ }^{1} \mathrm{~K}$. Suzuki

${ }^{1}$ Cátedra de Tecnología y Sanidad de los Alimentos, Facultad de Ciencias Veterinarias, Universidad Nacional de La Plata, Argentina

${ }^{2}$ Cátedra de Microbiología, Facultad de Ciencias Veterinarias, Universidad Nacional de Asunción, Paraguay ${ }^{3}$ Departamento de Bacteriología y Micología, Laboratorio Central de Salud Pública, Ministerio de Salud Pública y Bienestar Social, Paraguay

Corresponding Author: K Suzuki, Provetsur, Facultad de Ciencias Veterinarias, Universidad Nacional de La Plata, La Plata, B1900AVW, Argentina Tel/Fax: +542214253276

\begin{abstract}
The objective of this study was to (1) estimate frequencies of Salmonella enterica in growing pigs and (2) investigate farmers' perception and practices towards pig farming in Central Department, Paraguay. Twelve out of 19 districts in the department were selected and 33 farms with growers in the selected districts were recruited. Questionnaire interviews for each study farm, in combination with faecal sample collections ( $\mathrm{n}=30$ per farm), which were microbiologically examined and visual inspection of pig production facilities were performed between March and November 2009. A questionnaire was designed to obtain basic information of a farm such as the number of pigs owned and some selected farming management practices. Faecal samples were used for Salmonella isolation, using buffered peptone water to have salmonellae pre-enriched, followed by xylose lysine tergitol 4 agar and brilliant green sulfapyridine agar. Suspect colonies were biochemically tested by triple sugar iron agar in combination with lysine iron agar to confirm the identity. The true frequency probability and associated 95\% Bayesian credible intervals (95\% BCI) were computed via the Gibbs sampler, a Markov chain Monte Carlo technique. Overall, 18\% (95\% BCI: 8-31\%) of the tested 1000 faecal samples were classified as positive for Salmonella enterica. All the study farms had at least one positive sample for Salmonella enterica (frequency range: 3-60\%). Apparent prevalence at farm-level was therefore 100\% (one-sided $97.5 \%$ confidence limit: 89\%). Twenty-eight different serovars for Salmonella enterica were found. Based on increase the number of study districts, farms as well as animals in combination with improvement of sampling methods, possible spatial differences and risk factors/indicators should be clarified by further investigations.
\end{abstract}

Key words: Growers, salmonellosis, serovar, South America, swine

\section{INTRODUCTION}

Salmonellosis is seen worldwide and in all animals (Ayana and Surekha, 2008). The incidence has increased with the intensification of livestock production (Malkawi and Gharaibeh, 2004). The clinically normal carrier animal is a serious problem in all host species (Mohammad et al., 2006). The disease is caused by many species of salmonellae and characterized clinically by one or more of three major syndromes; septicemia, acute enteritis and chronic enteritis (Asghar et al., 2002). 
Chronic enteritis may develop in growing pigs (Mohammad et al., 2006). The incidence of human salmonellosis has increased in recent years and animals have been implicated as the main reservoir (Owlia et al., 2007). Transmission to humans occurs via polluted drinking water and foods; pork meat is one of particularly important sources of infection (Wales et al., 2009). Faeces of infected animals can contaminate feed and water, fresh and processed meats from slaughterhouses (Prendergast et al., 2008). The organisms may remain alive for months in wet, warm areas such as in feeder pig barns or in water dugouts (Owlia et al., 2007). Wildlife such as rodents are also sources of infection (Prendergast et al., 2008; Wales et al., 2009). The prevalence of infection differs amongst species and countries and is much higher than the incidence of clinical disease, which is frequently caused by stressful situations such as sudden deprivation of feed, parturition, drought, crowding, transportation and the administration of some drugs (Asghar et al., 2002; Prendergast et al., 2008; Wales et al., 2009). In Paraguay, field investigations to estimate frequencies of Salmonella enterica in pig populations were not previously implemented, to the best of the authors' knowledge. The objective of this study was to (1) estimate frequencies of Salmonella enterica in growing pigs and (2) investigate farmers' perception and practices tow ards pig farming in Central Department, Paraguay.

\section{MATERIALS AND METHODS}

This study was conducted between March and November 2009. Paraguay is located in the centre of South America and consists of 17 departments and one capital district. Paraguay has a pig population of 1.7 million and a pork meat production of 168,000 tonnes per year (FAO, 2010). The study area Central Department has 19 districts. Twelve out of the 19 districts were selected and 33 farms growing pigs in the selected districts were recruited. The required sample size of 1000 in total from a pig population of 1.7 million was sufficient to obtain a 95\% Confidence Interval (CI) with a desired precision of $\pm 3 \%$ when the estimated frequency was $50 \%$ (Hintze, 2008). The number of faecal samples of 30 at each farm was assigned by available financial, human and material means. The field study consisted of data collection through questionnaire interviews for each study farm, in combination with faecal sample collections and visual inspection of pig production facilities to find room of improvement. A questionnaire was designed to obtain basic information of a farm such as the number of pigs owned, some selected farming practices and farmers' perception for pig farming. Faecal samples were used for diagnostic tests. For Salmonella isolation, buffered peptone water was used to have salmonellae pre-enriched, followed by use of xylose lysine tergitol 4 agar (XLT4) and brilliant green sulfapyridine agar (BGS). Suspect colonies were biochemically tested by Triple Sugar Iron (TSI) agar in combination with Lysine Iron Agar (LIA) to confirm the identity, according to the USDA and OIE's recommendations (FSIS-USDA, 2008; OIE, 2009). Data were entered into a database using the Base in the OpenOffice.org software version 3.2.1 (Sun Microsystems, Santa Clara, CA, USA). A random effects hierarchical model was fitted at the first level using Bayesian methods which facilitate the estimation procedure. The number of test positive samples $r_{i}$ for individual study district were modelled as a binary response variable with True Frequency $(\mathrm{TF})$ probability $\mathrm{p}_{\mathrm{i}}$ :

$$
\mathrm{r}_{\mathrm{i}} \sim \operatorname{Binomial}\left(\mathrm{p}_{\mathrm{i}}, \mathrm{n}_{\mathrm{i}}\right)
$$

At the second level of the hierarchy, the model was to assume that percentage of test positive (apparent frequency; AF) were alike in some way. This was equal to specifying a random effects model for the TF probability $\mathrm{p}_{\mathrm{i}}$ as follows. They were assumed to be drawn from a common Normal population distribution: 
Res. J. Microbiol., 6(2): 159-165, 2011

$$
\begin{gathered}
\operatorname{logit}\left(p_{i}\right)=b_{i} \\
b_{i} \sim \operatorname{Normal}(\mu, \tau)
\end{gathered}
$$

The TF probability and associated $95 \%$ Bayesian credible intervals (95\% BCI) were computed via the Gibbs sampler, a Markov Chain Monte Carlo (MCMC) technique, which was implemented using WinBUGS software (Lunn et al., 2000). The exponential of these TF probabilities was taken to obtain overall frequency estimates (Freq) and their 95\% BCIs:

$$
\begin{gathered}
\text { Freq }=\frac{\exp (\mu)}{(1+\exp (\mu))} \\
\mu \sim \operatorname{Normal}(0.0,1.0 \mathrm{E}-6)
\end{gathered}
$$

Results presented here were based on multiple runs of length 100,000 following a burn-in of 10,000 iterations to achieve convergence.

\section{RESULTS}

Twelve different districts were selected (Fig. 1) and 33 farms were recruited (median number of the farms per district: two, range: one to eight). The study farms had 10,619 pigs in total at the visit (equivalent to $0.6 \%$ of the total pig population in Paraguay). The number of pigs reared varied

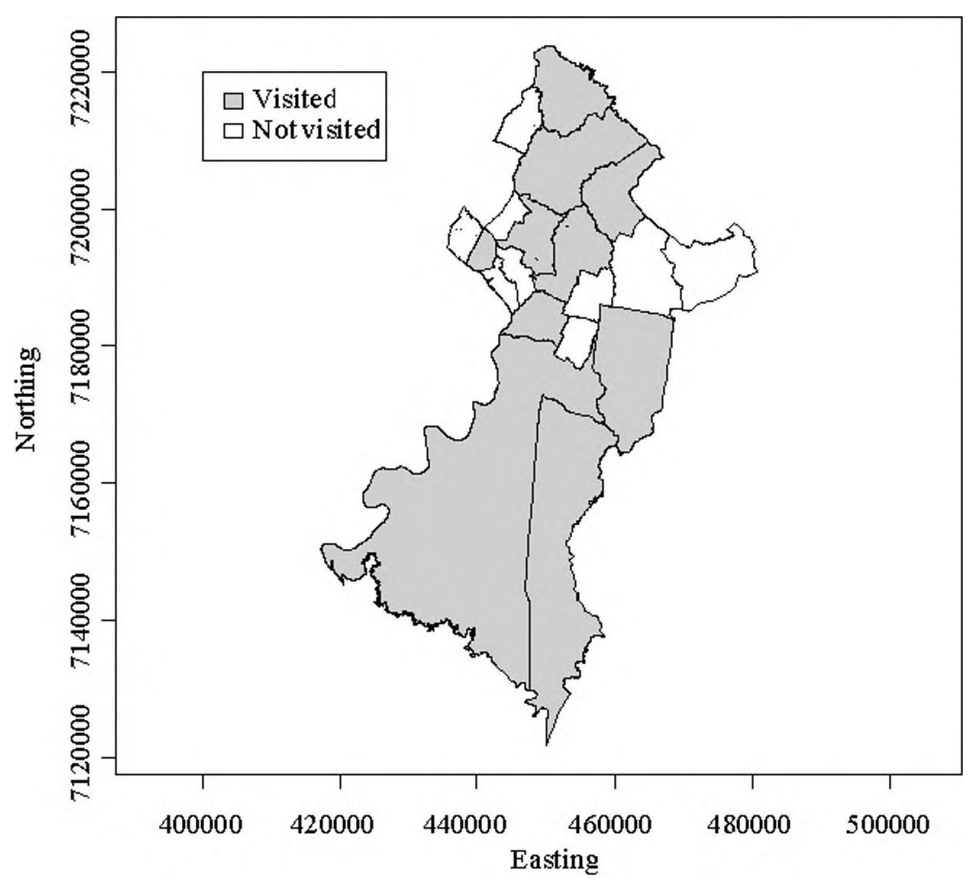

Fig. 1: A map showing the districts visited, for the field study of Salmonella enterica in growing pigs, in all the 19 administrative districts of Central Department, Paraguay 
between the study farms (range: 140-500, median: 345). Estimated median body weight at shipment for slaughter was $60 \mathrm{~kg}$ (range: $45-75 \mathrm{~kg}$ ). By visual inspection, $42 \%$ of the farms were found room for improvement in production facilities or not installing correct facilities. Forty-five percent of them had an incomplete sanitary plan for pig production or not having any plan. Twelve and three percent of them did not implement appropriate feed and water control and pest control, respectively. Half of them had an incomplete disinfection plan for the facilities or not having any plan. Twenty-four percent of them fed pigs leftover meals. Thirty-three and $20 \%$ of them owned animals other than pigs and let them free, respectively. Forty-five percent of them responded that their pigs showed possible symptoms caused by Salmonella infection in the last six months. Thirty percent of them gave medical treatment (e.g., antibiotics) to sick pigs. Thirty percent of them also experienced death of the sick pigs during the same period. In total 1000 different faecal samples randomly collected from the study pigs, on the basis of different sample sizes per district (range: 30-250, median: 60), were microbiologically examined. All the study farms had at least one positive sample for Salmonella enterica (frequency range: 3-60\%). Apparent prevalence at farm-level was therefore 100\% (one-sided $97.5 \%$ confidence limit: 89\%). Table 1 shows estimated frequencies of Salmonella enterica in study pigs. With regard to adjusted frequencies, all AF point estimates were well within the Bayesian credible intervals. TF point estimates varied between districts (range: 5-57\%). Overall, $18 \%$ (95\% BCI: 8-31\%) of the tested 1000 faecal samples were classified as positive for Salmonella enterica. Twenty-eight different serovars for Salmonella enterica were found. The most predominant serovar was $S$. typhimurium, followed by $S$. schwarzengrund and $S$. derby. Table 2 represents observed frequencies of predominant serovars for Salmonella enterica identified in the 1000 samples. Serovars with frequency of less than $0.5 \%$ include $S$. adamstua, $S$. javiana, $S$. mbandaka, $S$. bredeney, $S$. infantis, $S$. newport, $S$. albany, $S$. enteritidis, $S$. give, $S$. livingstone, $S$. sandiego, $S$. senftenberg, $S$. tennessee and $S$. westhampton in descending order of frequency. Besides Salmonella sp., finds frequency of 3.1\% (95\% CI: 2.1-4.4\%).

Table 1: Estimated frequencies of Salmonella enterica in growing pigs in Central Department, Paraguay

\section{Frequencies (\%)}

\begin{tabular}{|c|c|c|c|c|c|}
\hline & & & & & \\
\hline & & & & $95 \% \mathrm{BC}$ & \\
\hline District & $\mathrm{n}$ & $\mathrm{AF}$ & $\mathrm{TF}$ & Lower & Upper \\
\hline Areguá & 90 & 8 & 8 & 4 & 15 \\
\hline Capiatá & 250 & 20 & 20 & 15 & 25 \\
\hline Itá & 60 & 23 & 23 & 14 & 34 \\
\hline Limpio & 90 & 4 & 5 & 2 & 11 \\
\hline Luque & 60 & 3 & 5 & 1 & 11 \\
\hline Nueva Italia & 30 & 7 & 9 & 2 & 20 \\
\hline San Lorenzo & 90 & 14 & 15 & 8 & 22 \\
\hline Tablada & 210 & 24 & 24 & 18 & 30 \\
\hline Thompson & 30 & 60 & 57 & 38 & 72 \\
\hline Villa Elisa & 30 & 37 & 34 & 20 & 51 \\
\hline Villeta & 30 & 53 & 50 & 33 & 67 \\
\hline Ypané & 30 & 10 & 11 & 3 & 23 \\
\hline
\end{tabular}

n: No of faecal samples, AF: Apparent frequency, TF: True frequency, 95\% BCI: Bayesian credible interval 
Res. J. Microbiol., 6 (2): 159-165, 2011

Table 2: Frequencies of predominant serovars for Salmonella enterica identified in growing pigs in Central Department, Paraguay $(\mathrm{n}=1000)$

\begin{tabular}{lccc}
\hline & & $95 \%$ CI & Upper \\
Serovar & Frequencies (\%) & Lower & 4.4 \\
\hline Typhimurium & 3.1 & 2.1 & 3.7 \\
Schwarzengrund & 2.5 & 1.6 & 3.2 \\
Derby & 2.1 & 1.3 & 2.2 \\
Anatum & 1.3 & 0.7 & 2.2 \\
Cerro & 1.3 & 0.7 & 1.8 \\
Stanley & 1.0 & 0.5 & 1.4 \\
Rissen & 0.7 & 0.3 & 1.4 \\
Saintpaul & 0.7 & 0.3 & 1.2 \\
London & 0.5 & 0.2 & \\
\hline
\end{tabular}

\section{DISCUSSION}

The results presented here constitute the first departmental-wide survey carried out to estimate frequencies of Salmonella enterica in growing pigs in Paraguay. In summary, during the period between March and November 2009, the observed true frequencies at animal-level (5-57\%) and apparent prevalence at farm-level (100\%) in this study was similar to that reported by other authors in countries where Salmonella enterica infection is existed (Futagawa-Saito et al., 2008; Kishima et al., 2008; Merialdi et al., 2008; O'Connor et al., 2008; Dorn-In et al., 2009; Garcia-Feliz et al., 2009; Schwarz et al., 2009; Kim et al., 2010). In other countries in South America where Paraguay is located, pigs in Brazil had seroprevalence against Salmonella enterica of $74-83 \%$ at slaughterhouses (Schwarz et al., 2009). Other authors reported seroprevalence of Salmonella sp. of 38\% in Korea (Kim et al., 2010) and 94-100\% at herd-level in Italy (Merialdi et al., 2008). Others also reported prevalence of Salmonella enterica using faecal samples, such as 3\% at animal-level and 36\% at farm-level in Japan (Futagawa-Saito et al., 2008), 3\% at animal-level and $22 \%$ at farm-level also in Japan (Kishima et al., 2008), 63\% at animal-level in Thailand (Dorn-In et al., 2009) and 100\% at farm-level in 232 farms in Spain (Garcia-Feliz et al., 2009). O'Connor et al. (2008) reviewed farming management practices and characteristics associated with Salmonella prevalence in swine herds. The authors performed a questionnaire survey in this study to find a relationship between the positivity of Salmonella enterica at farm-level as outcome and such management practices as possible predictors using a logistic regression analysis, which failed due to the $100 \%$ prevalence at the level. Current sampling methods could be rise the uncertainty about the results of laboratory diagnostic tests. Rectal faecal samples collected from individually identified pigs randomly selected will give us more precise information. Based on increase the number of study districts, farms as well as animals in combination with improvement of sampling methods, possible spatial differences and risk factors/indicators should be clarified by further investigations.

\section{ACKNOWLEDGMENTS}

This study was carried out as part of the project for the capacity development for improvement of livestock hygiene in the southern part of South America through regional cooperation [commonly known as: Proyecto de desarrollo professional continuo para los veterinarios del Sur (PROVETSUR)], funded by the Japan International Cooperation Agency. 


\section{REFERENCES}

Asghar, U., Noor-us-Saba, A. Samad and A.A. Qazilbash, 2002. Identification, characterization and antibiotic susceptibility of salmonella and Shigella species isolated from blood and stool samples of patients visiting N. I. H, Islamabad. J. Med. Sci., 2: 85-88.

Ayana, N. and K. Surekha, 2008. Antimicrobial susceptibility pattern and characterization of ciprofloxacin resistant Salmonella enterica serovar typhi isolates in Kerala, South India. Res. J. Microbiol., 3: 654-660.

Dorn-In, S., R. Fries, P. Padungtod, M.N. Kyule and M.P.O. Baumann et al., 2009. A cross-sectional study of Salmonella in pre-slaughter pigs in a production compartment of northern Thailand. Prev. Vet. Med., 88: 15-23.

FAO, 2010. FAOSTAT. Food and Agriculture Organization of the United Nations, Rome, Italy.

FSIS-USDA, 2008. Isolation and Identification of Salmonella from Meat, Poultry and Egg Products. United States Department of Agriculture, Athens, GA.

Futagawa-Saito, K., S. Hiratsuka, M. Kamibeppu, T. Hirosawa, K. Oyabu and T. Fukuyasu, 2008. Salmonella in healthy pigs: Prevalence, serotype diversity and antimicrobial resistance observed during 1998-1999 and 2004-2005 in Japan. Epidemiol. Infect., 136: 1118-1123.

Garcia-Feliz, C., A. Carvajal, J.A. Collazos and P. Rubio, 2009. Herd-level risk factors for faecal shedding of Salmonella enterica in Spanish fattening pigs. Prev. Vet. Med., 91: 130-136.

Hintze, J., 2008. PASS 2008 Software. NCSS, Kaysville, UT, USA.

Kim, Y.H., Kwon, I.K. and J.H. Han, 2010. Seroprevalence of swine Salmonellosis in Korean swine herds. Korean J. Food Sci. Anim. Resour., 30: 62-65.

Kishima, M., I. Uchida, T. Namimatsu, T. Osumi and S. Takahashi et al., 2008. Nationwide surveillance of Salmonella in the faeces of pigs in Japan. Zoonoses Public Health, 55: 139-144.

Lunn, D.J., A. Thomas, N. Best and D. Spiegelhalter, 2000. WinBUGS - a Bayesian modelling framework: Concepts, structure and extensibility. Stat. Comput., 10: 325-337.

Malkawi, H.I. and R. Gharaibeh, 2004. Rapid and simultaneous identification of two Salmonella enterica serotypes, enteritidis and typhimurium from chicken and meat products by multiplex PCR. Biotechnology, 3: 44-48.

Merialdi, G., G. Barigazzi, P. Bonilauri, C. Tittarelli and M. Bonci, M. D'incau and M. Dottori, 2008. Longitudinal study of Salmonella infection in Italian farrow-to-finish swine herds. Zoonoses Public Health, 55: 222-226.

Mohammad, B., H. Mohagheghi Fard Amir and G. Roqiah, 2006. An investigation on contamination of poultries by Salmonella species in Zahedan (South-East Iran) during 2004. Res. J. Microbiol., 1: 463-466.

OIE, 2009. Salmonellosis, Manual of Diagnostic Tests and Vaccines for Terrestrial Animals. OIE Publishers, Paris.

O'Connor, A.M., Denagamage, T., Sargeant, J.M., Rajic, A. and J. McKean, 2008. Feeding management practices and feed characteristics associated with Salmonella prevalence in live and slaughtered market-weight finisher swine: A systematic review and summation of evidence from 1950 to 2005. Prev. Vet. Med., 87: 213-228.

Owlia, P., S. Leila, O. Fariba, R. Mohamad and B. Shahin, 2007. Evaluation of ceftriaxone releasing from microspheres based on starch against Salmonella sp. Biotechnology, 6: 597-600. 
Prendergast, D.M., S.J. Duggan, S. Fanning, M. Cormican and U. Gonzales-Barron et al., 2008. Prevalence and numbers of Salmonella sp. and enterobacteriaceae on pork cuts in abattoirs in the republic of Ireland. J. Applied Microbiol., 105: 1209-1219.

Schwarz, P., J. Calveira, A. Sella, M. Bessa, D. Barcellos and M. Cardoso, 2009. Salmonella enterica: Isolation and seroprevalence in swines slaughtered in Rio Grande do Sul. Arq. Bras. Med. Vet. Zootec., 61: 1028-1034.

Wales, A.D., I.M. McLaren, S. Bedford, J.J. Carrique-Mas, A.J. Cook and R.H. Davies, 2009. Longitudinal survey of the occurrence of Salmonella in pigs and the environment of nucleus breeder and multiplier pig herds in England. Vet. Rec., 165: 648-657. 